\title{
Research of Optimization Algorithms used in MIMO-OFDM Systems
}

\author{
Shaik Zubiya Hashmi, M. Vijaya Lakshmi
}

\begin{abstract}
Orthogonal Frequency Division Multiplexing (OFDM) technology is used to split large amount of data into several parallel narrowband channels with different frequencies orthogonally such that interference is reduced. Multiple Input Multiple Output (MIMO) technology uses diversity technique such that capacity of the system and data throughput can be improved. Thereby combining both the technologies as MIMOOFDM achieves great spectral efficiency and it is the most advanced technology in broadband wireless communication. The channel estimation techniques like Leaşt Square Estimation (LSE) algorithm is used to estimate the channel and the performance of MIMO-OFDM system is evaluated on the basis of Bit Error Rate (BER) and MeanSquare Error (MSE) by using MATLAB simulation. Further enhancement can be achieved by applying optimization algorithms, in this paper to find the optimum solution Particle Swarm Optimization Algorithm (PSO) is utilized when the pilots are placed randomly. Simulation outcome show that PSO algorithm outperforms the LSE when random pilots are used for MIMO-OFDM systems.
\end{abstract}

Keywords: “ Bit Error Rate”(BER), “Least Square Estimation”(LSE), “Mean Square Error”(MSE), “Multiple Input Multiple Output-Orthogonal Frequency Division Multiplexing”(MIMO-OFDM), “Particle Swarm optimization" (PSO)

\section{INTRODUCTION}

OFDM technology is a multicarrier system in which the signals are placed orthogonal to each other and transmitted over a narrowband channel with different frequencies so that the system can transmit large amount of data streams without interference thereby accommodating large number of users. Also by using OFDM technology crosstalk can be reduced. Another technology MIMO is used in modern communication systems in which diversity technique is implemented where two or more antennas are used both at the transmitting side and receiving side such that fading problems can reduced and also channel capacity and data throughput can be improved. So, by considering the advantages of both the technologies MIMO-OFDM technology is implemented and is used in $4 \mathrm{G}$ and $5 \dot{\mathrm{G}}$ broadband wireless communications.

In wireless communications "Channel State information" (CSI) is the most important parameter. This information helps to know about the propagation of the signal through the channel also describes about the distortion occurred and the delay caused by the signal from the transmitter to the

Revised Manuscript Received on 14 August, 2019.

ShaikZubiyaHashmi, M.tech Student, Assistant Professor, Department of Electronics and Telematics, G. Narayanamma Institute of Technology and Sciences (for women), Hyderabad, Telangana, India.(Email: zubiyahashmi.s@gmail.com)

M.Vijaya Lakshmi, M.tech Student, Assistant Professor, Department of Electronics and Telematics, G. Narayanamma Institute of Technology and Sciences (for women), Hyderabad, Telangana, India.(Email: vijayap03@gmail.com) receiver. The estimation of channel is based on some known information, which is known at the tranşmitter and the receiver. This method of estimating the channel helps to reconstruct the signal at the receiver end. Generally, the channel can be estimated by the usage of pilot symbols known both to the transmitter and the receiver, which employs different interpolation techniques such that the channel response of the subcarriersbetween the pilots can be estimated. Training signal or the training signals along with the data signals can be used for the estimation of the channel. Different channel estimation techniques like Blind channel estimation, semi-blind channel estimation, Training symbol based channel estimation are used. Blindchannel estimation scheme uses the properties of the received signals and the channel is estimated without the usage of the pilot symbols. Blind channel estimation technique has the benefit of less overhead but needs large number of received signals to reconstruct the signal. Semi-blind channel estimation scheme can improve the accuracy of the channel estimation by not only using the training or pilot symbols but also by using the unknown data symbols. This technique uses the detected or reconstructed signal as the feedback system to track the channel also uses that detected signal as the reference signal for the upcoming data. Training symbol based channel estimation provides good performance but the transmission efficiencies are reduced due to overhead of the "pilot symbols" which are transmitted along with the data symbols. When training symbols are used LS and MMSE techniques are used for the channel estimation thereby improving the system performance by minimizing the BER.

In Training symbol based channel estimation insertion of pilots is the important factor. So, the allocation of pilots can be done in different ways such as "Block type", "Comb type" and "Lattice type". In Block type pilot scheme, the transmitted symbols along with the pilots are transmitted using all the subcarrier from the transmitter. Here time domain interpolation is done. In Comb type pilot arrangement each transmitted symbols has the pilot tones at periodically placed subcarriers. Here frequency domain interpolation is done. In Lattice type of pilot arrangement both the time and frequency axis is used and by inserting pilots along the time and frequency axes in the given symbol period. Here both time and frequency interpolation is done. When orthogonal pilot allocation is made then these schemes can be useful but if random pilot allocation techniques are practiced the finding the optimum solution can be difficult. So, we utilize optimization algorithms. 
Optimization is the process to design a good system with the objective to minimize the cost of production or to maximize the efficiency of production. The procedure of optimization algorithms is to execute repeatedly by comparing different solutions till the best satişfactory solution is obtained.

In this paper the distorted signal in the system is estimated at the received side by using training signals or pilot signals. The optimization technique is applied to the random pilot signals and is compared with the fixed or orthogonal pilot signals to which the LSE algorithm is applied.

This paper is structured as follows, section I gives concise introduction of the MIMO-OFDM system and the usage of algorithms to approximate the channel. Section II tells about the channel Estimation mainly about the (LSE) and (MMSE) algorithms. Section III gives brief introduction about different optimization algorithms. Section IV explains about the Particle Swarm Optimization algorithm and also by using flow chart. Section V shows the simulation results. Section VI concludes the paper.

\section{CHANNEL ESTIMATION}

In communications the channel estimation is an important portion. The algorithms for channel estimation are to be selected in such a way that they satisfy both the time domain and frequency domain characteristics. Therefore Channel estimation and Optimization techniques are most essential works in MIMO-OFDM.

Algorithms like LSE, MMSE are used to reduce error rate in the MMO-OFDM system. Also algorithms like "Genetic Algorithm" (GA), "Evolutionary Programming" (EP), "Particle Swarm Optimization" (PSO), “Artificial Bee Colony" (ABC) and "Cuckoo Search"(CS) algorithms are used with the objective to minimize errors and thereby improving the performance of the system. The MIMOOFDM system is represented as

Where

$$
Y=H X+N
$$

$$
\begin{aligned}
& \mathrm{Y}=\text { Output Matrix }, \\
& \mathrm{X}=\text { Input Matrix } \\
& \mathrm{H}=\text { Channel Matrix }, \\
& \mathrm{N}=\text { AWGN Noise Matrix } .
\end{aligned}
$$

The LS estimate helps to calculate the channel estimate $\hat{H}$ to reduce the cost function as

$$
\begin{gathered}
J_{c f}(\hat{\mathrm{H}})=\|Y-X \hat{\mathrm{H}}\|^{2} \\
=(Y-X \hat{\mathrm{H}})^{H}(Y-X \hat{\mathrm{H}}) \\
=Y^{H} Y-Y^{H} X \hat{\mathrm{H}}-\hat{\mathrm{H}}^{H} X^{H} Y+\hat{\mathrm{H}}^{H} X^{H} X Y
\end{gathered}
$$

To make $\hat{H}$ zero assume derivative function as

$$
\frac{\partial \operatorname{Jcf}(\hat{\mathrm{H}})}{\partial \hat{\mathrm{H}}}=-2\left(X^{H} Y\right)^{*}+2\left(X^{H} X \widehat{\mathrm{H}}\right)^{*}=0
$$

From the above equation $X^{H} X \widehat{\mathrm{H}}=X^{H} Y$, therefor the solution to the channel matrix is give as

$$
\hat{\mathrm{H}}_{L S}=\left(X^{H} X\right)^{-1} X^{H} Y=X^{-1} Y
$$

Where $\hat{\mathrm{H}}_{L S}$ is the LS channel Estimate and the when $\mathrm{k}$ subcarriers are considered the $\widehat{\mathrm{H}}_{L S}(k)$ is given as

$$
\widehat{\mathrm{H}}_{L S}(k)=\frac{Y(k)}{X(K)}
$$

In MIMO-OFDM system Channel State Information (CSI) is estimated by using Least Square Error algorithm , According to that the channel matrix $\hat{\mathrm{H}}_{L S}$ is given as

$$
\hat{\mathrm{H}}_{L S}=X^{-1} Y
$$

CSI is estimated by using the MMSE the channel estimate matrix is given by $\mathrm{H}_{\text {MMSE. }}$ MMSE channel estimator uses the weight matrix $v$ which is given as $\hat{\mathrm{H}} \triangleq v \tilde{H}$.Mean Square Error (MSE) of the channel to be estimated is given as

$$
\operatorname{MSE}(\hat{\mathrm{H}})=E\left\{\left\|e^{2}\right\|\right\}=e\left\{\|H-\hat{\mathrm{H}}\|^{2}\right\}
$$

By using the weight matrix the channel can be estimated accurately. The weight vector or matrix can also be used for updating the symbols received thereby minimizing the MSE at the receiver side. As the pilot symbols are fixed or orthogonal to each other the error vector ' $e$ ' for estimation is given as

$\mathrm{e}=\mathrm{H}-\hat{\mathrm{H}}$ which is orthogonal to $\tilde{\mathrm{H}}$

$$
\begin{aligned}
E\{e \hat{\mathrm{H}} H\} & =E\{(H-\hat{\mathrm{H}}) H H\} \\
& =E\left\{E\left(H-\mathrm{v} H^{\sim}\right) \hat{\mathrm{H}} H\right\} \\
& =E\{H \hat{\mathrm{H}} H\}-\mathrm{v} E\{H \hat{\mathrm{H}} H\} \\
& =r H \hat{\mathrm{H}}-\mathrm{v} r H \hat{\mathrm{H}}=0
\end{aligned}
$$

Where $\mathrm{r}_{\mathrm{H} \mathrm{H}^{\sim}}$ represents the cross corelation of the $\square \square \square$ matrix

$$
\hat{\mathrm{H}}=X^{-1} Y=H+X^{-1} Z
$$

Now the equation for the weight vector is given as

$$
\mathrm{v}=r H H r H H-1
$$

The auto correlation matrix is given as

$$
r_{\tilde{\mathrm{H}} \tilde{\mathrm{H}}}=E\left\{\mathrm{H} \mathrm{H}^{\mathrm{H}}\right\}+\frac{\sigma_{Z}^{2}}{\sigma_{X}{ }^{2}} I
$$

Therefore the MMSE channel estimate equation is given as

$$
\begin{gathered}
H=\mathrm{v} H=r H H r H H-1 H \\
H M M S E=r H H\left(r H H+\frac{\sigma_{Z}^{2}}{\sigma_{X}{ }^{2}} I\right)-1 H
\end{gathered}
$$

The above equations of LSE $\mathrm{H}_{\mathrm{LS}}$ and MMSE $\mathrm{H}_{\text {MMSE }}$ gives the channel estimate and the error can be calculated by the channel coefficients.

\section{III.OPTIMIZATION ALGORITHMS}

Optimization techniques are evolved by the inspiration of biological methods like mutation, crossover, inheritance and selection. Optimization is a process that attempts to optimize i.e., to maximize or minimize the objective function by using mathematical steps. It is also called as mathematical programming. An optimization algorithm is a method in which the optimum or satisfactory solution is found by iteratively comparing various solutions. Therefore the purpose of optimization is to find the "best" solution and design the system relative to the given constraints.

\subsection{Genetic Algorithm:}

Genetic Algorithm (GA) [1] is an adaptive search algorithm used for finding the minimum cost function and to maximize the fitness value. Genetic algorithm is based on optimization technique inspired by operators such as mutation, cross over, selection process to generate optimum solutions. This is also a type of Evolutionary algorithm 
where the solution can be found when there is more number of variables. So, GA provides optimum solution if large number of variables are used in the MIMO-OFDM system. This algorithm provides optimum solution to the problem and is one of the bestalgorithms and is used in many applications like in mobile communication systems.

GA has three components mainly reproduction, mutation and crossover these are used to solve the search and optimization problem and find the optimum solution .The best channel is selected by calculating the fitness value. The fitness equation is given as

$$
\text { fitness }=\left(\frac{H-H_{G A}}{H}\right)^{2}
$$

The channel Estimation by using this GA is done by using the simulation parameters. The channel estimation parameters can be evaluated by using Genetic Algorithm.

\subsection{Evolutionary Programming Algorithm}

Evolutionary Programming (EP) is an Optimization Algorithm also an Evolutionary algorithm like Genetic algorithm (GA). In this algorithm only Mutation is considered and the fitness value is calculated. In this algorithm the population is considered. This algorithm is used to improve the fitness value through mutation and recombination. Therefore the fitness value for EP algorithm

$$
\text { fitness }=\left(\frac{H-H_{E P}}{H}\right)^{2}
$$

Evolutionary Programming Algorithm is a significant optimization technique and Parallel process with low complexity and takes less computational time. Like Genetic algorithm this Evolutionary Programming algorithm also can be used for channel estimation of MIMO-OFDM system but gives global optimal solution with the probability of one giving better results when compared to other optimization techniques.

\subsection{Artificial Bee Colony Algorithm}

The ArtificialBee Colony Algorithm (ABC) [4] is one of the recent algorithms motivated by the intelligent behavior of the honey bees. This Artificial Bee Colony algorithm is population based search algorithm which is used as an optimization tool. In this algorithm the individuals are called food positions which are changed according to the time these positions are helpful in finding the optimum solution. In this $\mathrm{ABC}$ algorithm, the colony of artificial bees has three groups they are Employed bees, onlookers and scouts. The bee which makes decisions to choose food source is called onlookers. The bee which visits the food source is called an Emp1oyed bee and the bee that performs random search to discover new sources is called scout.The fitness function is calculated as

$$
f i t_{i}=\frac{1}{f i}
$$

Where fit $t_{i}$ is the fitness and the fi is the cost function of the channel estimation. The optimum value obtained by $\mathrm{ABC}$ can be used for channel estimation.

\subsection{Cuckoo Search Algorithm}

Cuckoosearchalgorithm [3] is also one of the optimization algorithms. This is introduced to enhance the performance is calculated as follows

of the MIMO-OFDM system. This algorithm is used to obtain the most desired parameter and the undesired parameter can be obtained less. The aim of this Cuckoo search algorithm is to decrease the error and maximize the transmission speed. Channel estimation is performed by using this Cuckoo search algorithm.

\section{PARTICLE SWARM OPTIMIZATION ALGORITHM}

Particle Swarm Optimization [2] is a meta heuristic optimization algorithm which is inspired by school of fish, flock of birds such that to adapt to the surroundings and sharing the information or data. This is population based search algorithm. Particle Swarm Optimization method is used to design pilots. This algorithm is used when large number of random data is used in the system. In MIMOOFDM system random pilots are used in such case this algorithm can be used to find the optimum solution. So, in the MIMO-OFDM system each subcarrier can be considered as particle. Each particle memorizes the best position by searching the search space. The best fitness value position is set as pbest and one the best of all the particles in the population considered is set as global best i.e, gbest but the pbest and gbest have different positions and these positions and their directions are used to find the optimum value. This is compared with orthogonal and random placements of the pilots. This is compared and evaluated in terms of BER and MSE.

PSO is a computational or optimization technique. This algorithm helps to optimize the problem to get the optimum solution by iterative process. The optimum solution obtained is used to identify the best channel. Normally, PSO consists of four stages namely, initializing the particle, evaluation function, updațing the initial particle, and terminaţion.

The realization of this algorithm is simple and also has fast convergence. In "Particle Swarm Optimization"(PSO) each particle is called as an agent and is placed in the search space to find the objectivefunction from the current location. Each particle in the search space searches for the best position by changing the velocity.

The inițialposition and velocity of the particles are generated randomly in the search space. The posițion and velocity of the particle ' $i$ ' are updated for every iteration or repetition with dimension ' $D$ '. The position vector of each particle ' $\mathrm{i}$ ' is given as

$$
x i=(x i 1, x i 2, \ldots \ldots x i D)
$$

and the velocity vector is given as

$$
v i=(v i 1, v i 2, \ldots \ldots . . v i D)
$$

where $\mathrm{D}$ is the dimension of the solutionspace.For each iteration the velocity and position of the particle can be calculated as follows

$$
\begin{array}{r}
\operatorname{vid}(t+1)=w \operatorname{vid}(t)+\operatorname{c1ri1}(t)(\text { pbestid }(t) \\
-x i d(t))+\operatorname{cri2}(t)(\operatorname{gbesti}(t) \\
-x i d(t))
\end{array}
$$

The previous best position of the particle is given as pbestid $=(p i 1, p i 2, \ldots \ldots$ piD $)$ and the best position of all the particles is given as gbestd $=(p 1, p 2, \ldots p D) . \mathrm{r}_{\mathrm{i}}{ }^{1}$ 
and $\mathrm{r}_{\mathrm{i}}^{2}$ are evenlydistribuțednumbers and lies in the interval $[1,0], \mathrm{c} 1$ and $\mathrm{c} 2$ are cognitive and social values and $\mathrm{w}$ is the inertia weights used to maintain the momentum of the particle. Global search is done when the inertia weight is large. Local search is done when the inertia weight is small. Therefore the inertia weights should be selected suitably to find the optimum solution. The inertia weight can be reduced linearly from $\mathrm{w}_{\max }$ to $\mathrm{w}_{\min }$ by using the formula

$$
w=w_{\text {max }}-\frac{w_{\max }-w_{\min }}{\text { iteration }_{\max }} \text { xiteration }
$$

Algorithm for Particle Swarm Optimization is given as:

Step 1: First the particles are initialized according to the number of subcarriers

Step 2 : The evaluation function or the objective function is to be calculated to find the best initial particle using ParticleSwarmOptimization algorithm.

$$
\text { Evaluation function }=\left(\frac{H-H_{P S O}}{H}\right)^{2}
$$

Where,

$\mathrm{H}$ is referencechannel matrix and

Hpso is channel matrix when PSO algorithm is used

Step 3 : The velocity and current position of the particle is updated. These are updated by the following equations,

$$
\begin{gathered}
V[]=w v[]+c 1 \operatorname{rand}()(\text { pbest }[]-\operatorname{present}[]) \\
+c 2 \operatorname{rand}()(\text { gbest }[]-\operatorname{present}[]) \\
\text { and } \\
\text { Present }[]=\operatorname{present}[]+v[]
\end{gathered}
$$

Where, $v[]$ is the particle velocity, present [] is the current particle, pbest [] and gbest[] are the local and global best particles, respectively, $\operatorname{rand}()$ is the random number between $[0,1]$, and $c 1, c 2$ are the learning factors

Step 4 : After the initial particle is updated the process is terminated. The best channel is estimated by the calculated evaluation function.

The best channel is estimated, by the channel estimators obtained by Least Square Estimation algorithm and ParticleSwarmOptimization is compared and the best channel is selected based on minimum error value. The estimated channel with minimum error value is selected as the better channel estimation. The PSO algorithm has many applications in signal processing, Antenna designing, Image and video analysis, Robotics.

PSOAlgorithm helps to improve the performance of the system. This algorithm is evaluated by simulation method and the results are compared with the LS Channel estimate.

The flow chart for the above Particle Swarm optimization algorithm is as shown below.

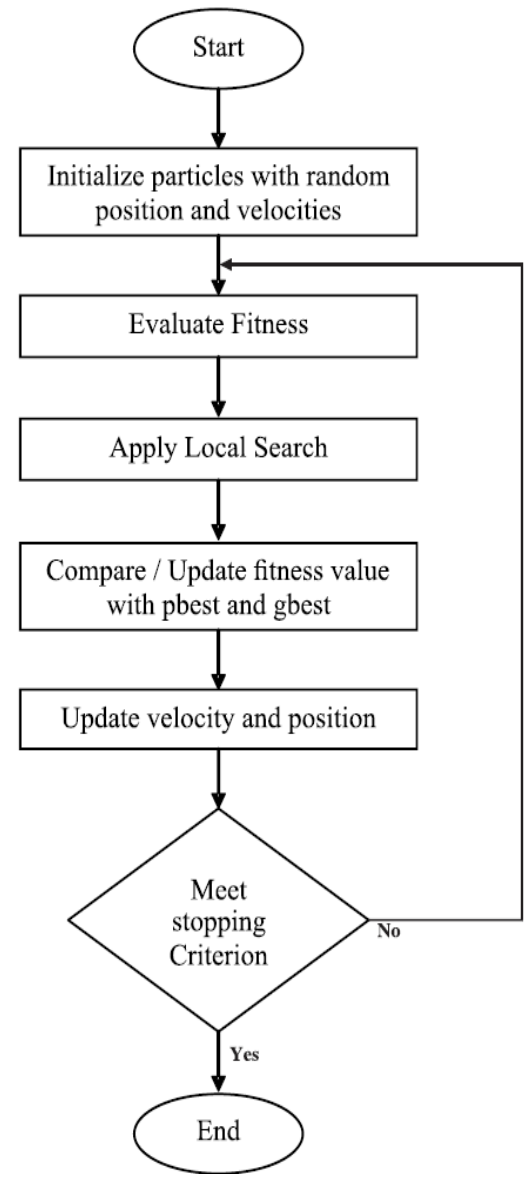

Fig 4.1: Flow Chart for PSO Algorithm

\section{V.SIMULATION RESULTS}

Simulation Parameters:

\begin{tabular}{|l|c|}
\hline Number of b1ocks & $\mathrm{Nb}=10$ \\
\hline $\begin{array}{l}\text { Length of OFDM } \\
\text { symbo1s }\end{array}$ & $\mathrm{K}=128$ \\
\hline Constellation order & $\mathrm{Mod}=4$ \\
\hline Constellation type & $\mathrm{PL}=64$ \\
\hline Pilot length & $\mathrm{L}=6$ \\
\hline $\begin{array}{l}\text { Channel length (No. } \\
\text { of channel taps) }\end{array}$ & \\
\hline
\end{tabular}

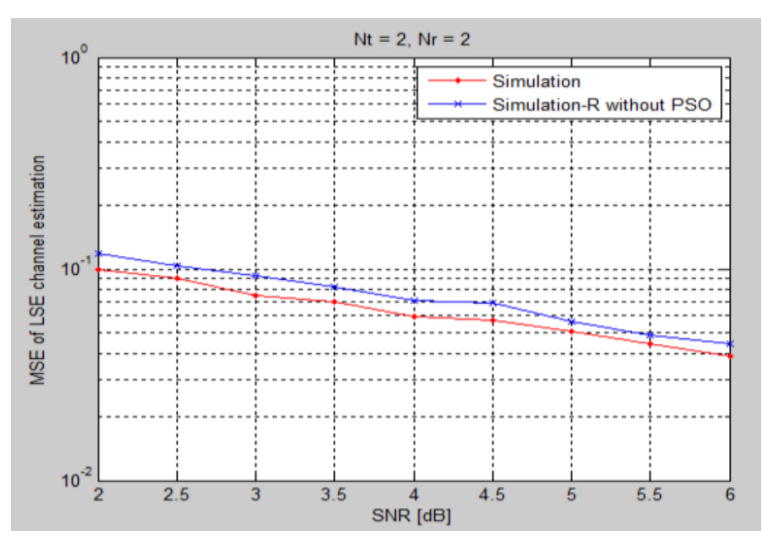

Fig 5.1: MSE vs SNR for Orthogonal and Random pilot allocation without using optimization algorithm 
Table 5.1: Comparison of MSE values for Orthogonal and Random pilots without PSO algorithm

\begin{tabular}{|c|c|c|}
\hline \multirow{2}{*}{ SNR(dB) } & \multicolumn{2}{|c|}{ Mean Square Error (MSE) } \\
\cline { 2 - 3 } & Orthogonal & Random \\
\hline 3 & 0.07481 & 0.09234 \\
\hline 4.5 & 0.05684 & 0.06952 \\
\hline 6 & 0.03878 & 0.04401 \\
\hline
\end{tabular}

Table 5.1 gives the information about the MSE values for the Orthogonal and Random pilots without optimization algorithm when different values of Signal to Noise Ratio (SNR) in $\mathrm{dB}$ are considered.

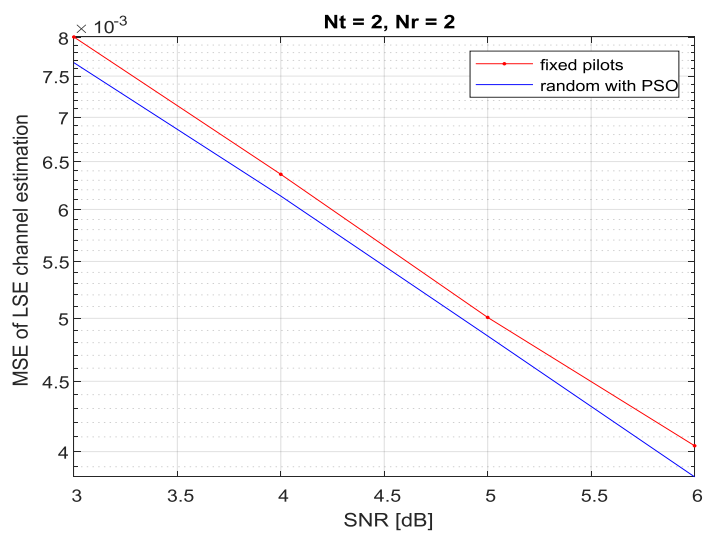

Fig 5.2: MSE vs SNR when PSO algorithm is applied to random pilots.

Table 5.2: Comparison table of MSE values for Orthogonal and Random Pilots when PSO algorithm is applied

\begin{tabular}{|c|c|c|}
\hline \multirow{2}{*}{ SNR(dB) } & \multicolumn{2}{|c|}{ Mean Square Error (MSE) } \\
\cline { 2 - 3 } & Orthogonal & Random \\
\hline 3 & 0.008 & 0.0077 \\
\hline 4.5 & 0.0056 & 0.0054 \\
\hline 6 & 0.00405 & 0.0038 \\
\hline
\end{tabular}

Table 5.2 gives MSE values of the Orthogonal and Random pilots when PSO algorithm is applied for the Random pilots when different SNR values are taken.

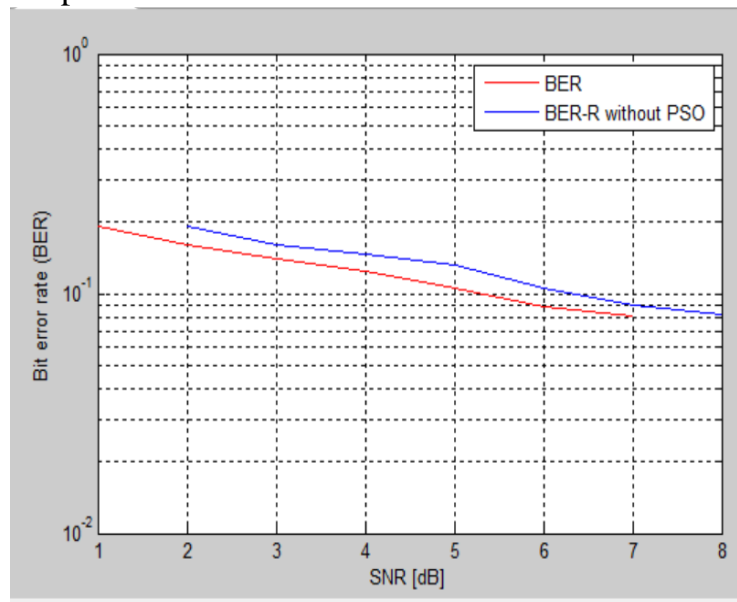

Fig 5.3: Figure represents BER vs SNR for Orthogonal and Random Pilots when Optimization algorithm is not applied.
Table 5.3: Comparison table of BER values for Orthogonal and Random pilots

\begin{tabular}{|c|c|c|}
\hline \multirow{2}{*}{ SNR(dB) } & \multicolumn{2}{|c|}{ Bit Error Rate (BER) } \\
\cline { 2 - 3 } & Orthogonal & Random \\
\hline 2 & 0.1656 & 0.1984 \\
\hline 4 & 0.1273 & 0.1513 \\
\hline 6 & 0.0838 & 0.1256 \\
\hline
\end{tabular}

Table 5.3 shows the values of BER for various SNR values when Orthogonal and Random pilots are considered without using optimization algorithm.

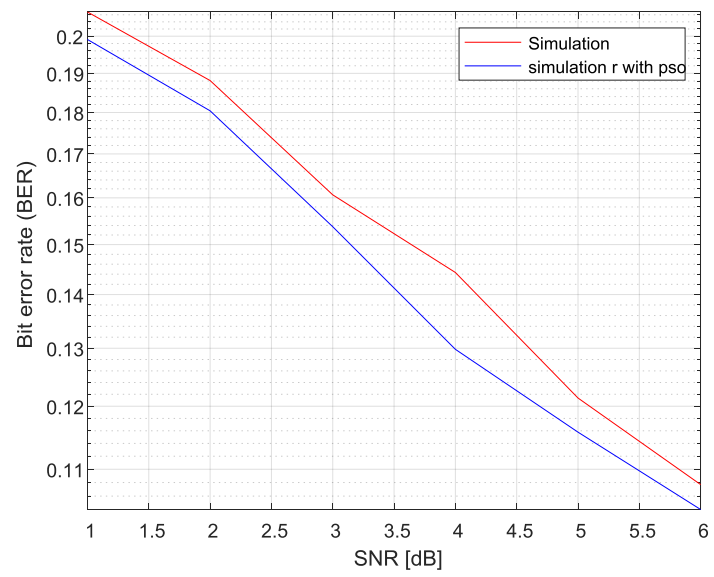

Fig 5.4:Plot for BER vs SNR when the PSO algorithm is applied for random pilots.

Table 5.4: Comparisonof BitErrorRate(BER) values for Orthogonal and Radom Pilots when PSO algorithm is considered.

\begin{tabular}{|c|c|c|}
\hline \multirow{2}{*}{ SNR(dB) } & \multicolumn{2}{|c|}{ Bit Error Rate(BER) } \\
\cline { 2 - 3 } & Orthogonal & Random \\
\hline 2 & 0.188 & 0.18 \\
\hline 4 & 0.144 & 0.13 \\
\hline 6 & 0.108 & 0.104 \\
\hline
\end{tabular}

Table 5.4 shows that the BER values of the Orthogonal and Random pilots when PSO algorithm is applied to the Random pilots at different values of SNR .

\section{CONCLUSION}

In this paper, different optimization algorithms are included and also traditional channel estimation algorithms like Least Square Estimate and Minimum Mean Square Error algorithms were also included. The pilot tones designed as orthogonal pilots and random pilots are calculated in terms of Bit Error Rate and MeanSquareError. In this paper, the channel is estimated by Least Square Estimate and by optimizing pilot by Particle Swarm Optimization. The simulation results show that the optimized pilot tones outperforms the Least Square Estimate algorithm. The "Particle SwarmOptimization" (PSO) algorithm used in this paper is easy to implement and has less complexity. The performance of MIMO-OFDM is

Published By

Blue Eyes Intelligence Engineering

\& Sciences Publication 
evaluated in terms of BER and MSE for orthogonal and random arrangement of the pilots in the system.

\section{REFERENCES}

1. Nitin Sharma, K.R. Anupama, "A Novel Genetic Algorithm for Adaptive Resource Allocation in MIMOOFDM systems" with Proportional Rate constraints. Wireless PersCommun (2011) 61:113-128 DOI 10.1007/s11277-010-0013-9

2. MuhammetNuriSeyman,NecmiTaspinar, "Particle Swarm Optimization for Pilot Tones Design in MIMOOFDM Systems" EURASIP Journal on Advances in Signal Processing 20112011:10 doi.org/10.1186/16876180-2011-10

3. Deepan Kishore Kumar, "Channel Estimation of MIMOOFDM System using Cuckoo Search Algorithm" , Published 2013

4. MuhammetNuriSeyman, NecmiTaspinar, " Pilot Tones Optimization Using Artificial Bee Colony Algorithm for MIMO-OFDM System" , Wireless Personal Communications July 2013, Volume 71, Issue 1, pp 151163

5. LJ CiminiJr, "Analysis and simulation of digital mobile channel using orthogonal frequency division multiplexing". IEEE Trans Commun.3(7):665-675 (1985)

6. H Sampath , S Talwar, "A fourth-generation MIMOOFDM broadband wireless systems: design performance and trial results". IEEE Commun Mag.40(9):143149(2002).doi:10.1109/MCOM.2002.103 1841

7. S Coleri, M Ergen, A Puri, A Bahai, "Channel estimation techniques based on pilot arrangement in OFDM systems". IEEE Trans Broadcast. 48(3):223229(2002).doi:10.1109/TBC.2002.804034

8. R Negi, J Cioffi, "Pilot tone selection for channel estimation in a mobile OFDM system". IEEE Trans ConsumElectron. 44(3):11221128(1998).doi:10.1109/30.713244

9. M Dong, L Tong, "Optimal design and placement of pilot symbols for channel estimation". IEEE Trans Signal Process. 50(12):3055-3068 (2002).doi:10.1109/TSP.2002.805504

10. X Cai, GB Giannakis, "Error probability minimizing pilots for OFDM with MPSK modulation over Rayleigh fading channels". IEEE Trans VehTechnol.53(1):146155 (2004). doi:10.1109/TVT.2003.819624

11. I Barhumi, G Leus, M Moonen, "Optimal training design for MIMO-OFDM systems in mobile wireless channels". IEEE Trans Signal Process.51(6):1615-1623 (2003). doi:10.1109/TSP.2003.811243

12. R.S.Ganesh, DrJ.Jayakumari, Akhila I.P "Channel Estimation Analysis in MIMO-OFDM Wireless Systems" icscen 2011 proceedings.

13. D Hu, L Yang, L He, Y Shi, "Optimal pilot sequence design for multiple input multiple output OFDM systems". IEEE Global Telecom Conference (GLOBECOM'05). (Saint Louis, USA, 2005), pp. 22602264 